\title{
Magnetic particles confined in a modulated channel: Structural transitions tunable by tilting a magnetic field
}

\author{
J. E. Galván-Moya, ${ }^{1, *}$ D. Lucena, ${ }^{1,2}$ W. P. Ferreira, ${ }^{2}$ and F. M. Peeters ${ }^{1,2, \dagger}$ \\ ${ }^{1}$ Department of Physics, University of Antwerp, Groenenborgerlaan 171, B-2020, Antwerp, Belgium \\ ${ }^{2}$ Departamento de Física, Universidade Federal do Ceará, Caixa Postal 6030, Campus do Pici, 60455-760 Fortaleza, Ceará, Brazil
}

(Received 20 December 2013; published 31 March 2014)

\begin{abstract}
The ground state of colloidal magnetic particles in a modulated channel are investigated as a function of the tilt angle of an applied magnetic field. The particles are confined by a parabolic potential in the transversal direction while in the axial direction a periodic substrate potential is present. By using Monte Carlo simulations, we construct a phase diagram for the different crystal structures as a function of the magnetic field orientation, strength of the modulated potential, and the commensurability factor of the system. Interestingly, we found first- and second-order phase transitions between different crystal structures, which can be manipulated by the orientation of the external magnetic field. A reentrant behavior is found between two- and four-chain configurations, with continuous second-order transitions. Novel configurations are found consisting of frozen solitons of defects. By changing the orientation and/or strength of the magnetic field and/or the strength and periodicity of the substrate potential, the system transits through different phases.
\end{abstract}

DOI: 10.1103/PhysRevE.89.032309

PACS number(s): 82.70.Dd, 52.27.Lw, 64.60.Cn, 81.30.-t

\section{INTRODUCTION}

Competitive interactions between particles are responsible for the formation of a large variety of complex structures in nature [1-3]. However, to take advantage of this aspect, the major challenge is to find a way to manipulate this formation process, in order to obtain the desired configuration through a correct choice of a set of parameters. Although this idea seems to be promising, there are many other obstacles to overcome.

Formation of Wigner crystals has been widely studied for a purely repulsive interaction between particles in quasi-onedimensional (Q1D) [4-6], two-dimensional (2D) [7,8], and three-dimensional (3D) $[9,10]$ systems. These studies showed a very rich set of ground-state configurations depending on the system properties, and a diverse kind of transition was found between them. However, the formation of complex structures increases dramatically when particles interact via competing interaction [11-14]. One of the most simple models is based on the dipole-dipole interaction $[15,16]$, which can be realized by an external field acting on a system of magnetic particles. The multiple crystalline structures that can be found [17,18], and the effects of external fields applied on these particles, which have been summarized in a recent minireview [19], indicate the promising future of these kind of systems.

Recent works showed the effect of a fixed and oscillating external magnetic field on the crystal structures [20,21], evidencing that the configuration of the system can be controlled by the orientation of the magnetic field. Numerical studies of these systems demonstrated the possibility of cluster formation $[22,23]$ as well as few-body bound states $[24,25]$ and local deformations in elongated dipolar gases [26]. Additionally, different transitions between Fermi liquid, solitons, and Wigner crystals have been predicted [27,28] as well as some tunable assemblies [29]. Also ground-state

\footnotetext{
*jesuseduardo.galvanmoya@uantwerpen.be

†francois.peeters@uantwerpen.be
}

configurations of 2D dipolar mixtures were investigated for different orientations of an external magnetic field by using a genetic algorithm [30-32], showing that the complexity of the particles arrangement increases with the anisotropy of the interparticle interaction.

The effect of complex potential landscapes on the structure of interacting colloidal particles was investigated in one dimension [33-35] and recently also in two dimensions [36]. A periodic potential has been proposed to control the arrangement of the particles [13,36,37], which is triggered by the commensurability $[38,39]$ between the periodicity of the potential and the average distance, i.e., the density, of the particles. Experimentally, it was found that a colloidal suspension, where the interaction between particles is negligible, exhibits a nontrivial dependence on the strength of the modulated potential [40]. This finding allows the possibility to use the modulated potential as an effective tunable parameter for a system of interacting particles.

In the present work the ground-state configuration of a system of magnetic particles confined by a parabolic Q1D channel on a periodic substrate potential is studied. Magnetic particles are aligned with an external tilted magnetic field and interacting through an anisotropic dipole-dipole potential [15], which depends on the orientation of the external field. We investigate how the orientation of the field allows us to tune the ground-state configuration of the system, modulating the competitive interaction between each pair of particles. The effect of the modulated potential is analyzed through its strength and the degree of commensurability of the system imposed by the periodicity of the substrate potential and the average interparticle distance, i.e., the density.

The present paper is organized as follows. In Sec. II the model system and the numerical methods are described. In Sec. III we present the results for the different ground-state transitions of the commensurate system, and the tuning between the different phases is realized by changing the orientation of the magnetic field and the external modulated potential. In Sec. IV the same analysis is presented for a noncommensurate system, and the effects due to the 


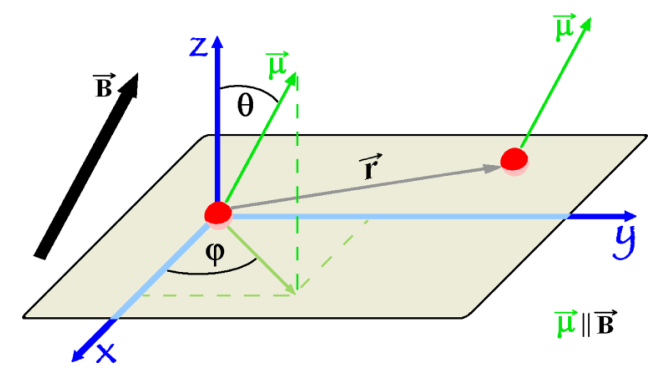

FIG. 1. (Color online) Schematic representation of two dipolar particles (red spheres). All particles are confined to the $x-y$ plane and the magnetic moment $\boldsymbol{\mu}$ of each one is aligned to the external magnetic field $\mathbf{B}$.

commensurability factor are explained. Our conclusions are given in Sec. V.

\section{MODEL SYSTEM}

In this work, we consider a system of $N$ identical interacting magnetic particles, which are allowed to move in the $x-y$ plane. The magnetic particles are confined by an external parabolic potential in the $y$ direction and a periodic substrate potential along the $x$ direction with period length $L$ and potential height $V$.

The system is subjected to an external and spatially homogeneous magnetic field $\mathbf{B}$, which induces a magnetic moment $\boldsymbol{\mu}$ to all particles, aligning them to $\mathbf{B}$. The magnetic field is tilted by an angle $\theta$ with respect to the $z$ axis, which is perpendicular to the plane of motion, and it forms an angle $\varphi$ with respect to the $x$ axis of the plane. This is schematically represented in Fig. 1.

At short distances the interaction between the colloidal particles is a hard-core repulsion, which defines the characteristic length (the diameter of each particle, $\sigma$ ) and the energy scale $(\varepsilon)$ of the system. The total energy of the system is given by

$$
\begin{aligned}
H= & \sum_{i=1}^{N}\left[\frac{1}{2} m \omega_{0}^{2} y_{i}^{2}-V \cos \left(\frac{2 \pi x_{i}}{L}\right)\right] \\
& +\sum_{i=1}^{N} \sum_{j>i}^{N}\left[4 \varepsilon\left(\frac{\sigma}{\left|\mathbf{r}_{i j}\right|}\right)^{12}+V_{\mathrm{dip}}\left(\mathbf{r}_{i j}, \boldsymbol{\mu}\right)\right],
\end{aligned}
$$

where $\mathbf{r}_{i j}=x_{i j} \hat{\mathbf{e}}_{\mathbf{x}}+y_{i j} \hat{\mathbf{e}}_{\mathbf{y}}$ represents the relative position between the $i$ th and the $j$ th particle and $V_{\text {dip }}\left(\mathbf{r}_{i j}, \boldsymbol{\mu}\right)$ is the dipole-dipole interaction $[15,16,24]$ given by

$$
V_{\mathrm{dip}}\left(\mathbf{r}_{i j}, \boldsymbol{\mu}\right)=\frac{1}{r_{i j}^{5}}\left[\left(\mu r_{i j}\right)^{2}-3\left(\boldsymbol{\mu} \cdot \mathbf{r}_{i j}\right)^{2}\right],
$$

where we assume that the magnetic moment of each particle $\boldsymbol{\mu}$ is aligned to $\mathbf{B}$. Equation (2) can be rewritten as follows

$$
\begin{aligned}
V_{\text {dip }}\left(\mathbf{r}_{i j}, \theta, \varphi\right)= & \frac{\mu^{2}\left[1-3 \sin ^{2} \theta \sin ^{2}(\varphi)\right]}{\left|\mathbf{r}_{i j}\right|^{3}} \\
& -\frac{3 \mu^{2} \sin ^{2} \theta}{\left|\mathbf{r}_{i j}\right|^{5}} x_{i j}\left[\cos (2 \varphi) x_{i j}+\sin (2 \varphi) y_{i j}\right] .
\end{aligned}
$$

A contour plot of the pairwise interaction of Eq. (3), is shown in Fig. 2 as a function of the relative $x$ and $y$ distances between two particles for different values of $\theta$, as specified in the figures, with fixed $\varphi$. The anisotropy of the dipole-dipole interaction (see Fig. 2) plays an important role in the determination of the many-particle ground-state configuration, as it will be discussed later, breaking the widely studied chainlike structure found for systems with isotropic pairwise interaction [4-6]. The anisotropy of the interaction is governed by the angles $\theta$ and $\varphi$, nevertheless, as shown in this work, the strength of the periodic potential can overcome
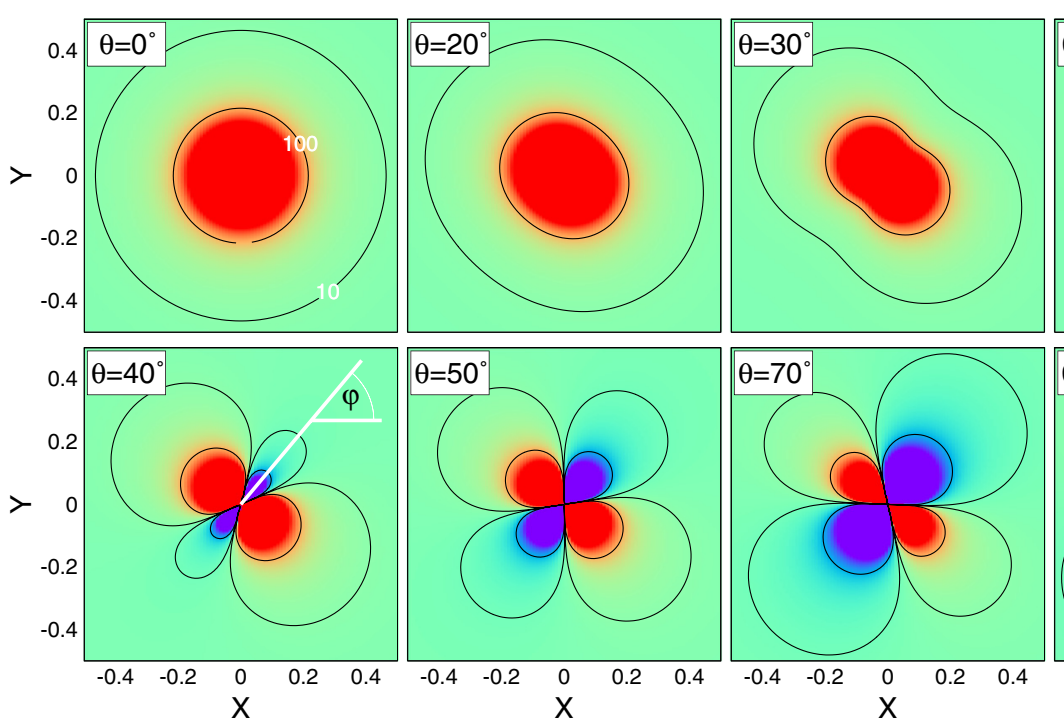

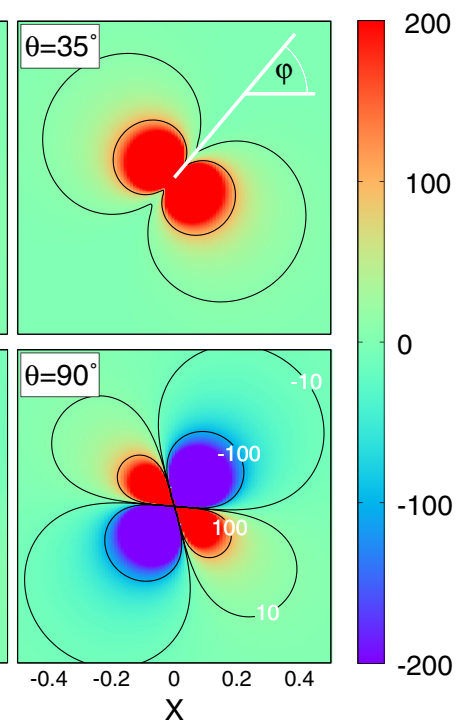

FIG. 2. (Color online) The dipole-dipole interaction as a function of the relative $(x, y)$ position between two particles, is plotted for different values of the angle $\theta$ between the magnetic field and the $z$ axis. The value of $\theta$ is specified in the figures, while the strength of the interaction is indicated by the color bar. The contour lines are plotted for different values of the interaction energy, as labeled in the first and last figures. The direction of $\mathbf{B}$ in the $x-y$ plane is indicated by the angle $\varphi$ in the top right figure, which was taken $\varphi=50^{\circ}$. 
the effect of the anisotropy, determining the many-particle configuration.

In dimensionless units, the total energy of Eq. (1) becomes

$$
\begin{aligned}
H= & \sum_{i=1}^{N}\left[v^{2} y_{i}^{2}-V_{0} \cos \left(\frac{2 \pi x_{i}}{L}\right)\right. \\
& +\sum_{j>i}^{N}\left\{\frac{4}{\left|\mathbf{r}_{i j}\right|^{12}}+\frac{\mu^{2}\left[1-3 \sin ^{2} \theta \sin ^{2}(\varphi)\right]}{\left|\mathbf{r}_{i j}\right|^{3}}\right. \\
& \left.\left.-\frac{3 \mu^{2} \sin ^{2} \theta}{\left|\mathbf{r}_{i j}\right|^{5}} x_{i j}\left[\cos (2 \varphi) x_{i j}+\sin (2 \varphi) y_{i j}\right]\right\}\right],
\end{aligned}
$$

where the energy and the distances are given in units of $\varepsilon$ and $\sigma$, respectively. The dimensionless confinement frequency is defined by $v^{2}=m \omega_{0}^{2} / 2 \varepsilon \sigma^{2}$ and the dimensionless strength of the substrate potential by $V_{0}=V / \varepsilon$, while the dimensionless magnetic moment of each particle can be redefined by $\boldsymbol{\mu} / \varepsilon \sigma^{3} \Rightarrow \boldsymbol{\mu}$.

We introduce the dimensionless linear density $\eta$ and the periodicity number $n$, defined respectively as the number of particles and the number of periods of the periodic potential per unit length along the $x$ direction. The presence of the periodic potential leads to the commensurability factor [38]

$$
p=\frac{N}{n}=\eta L,
$$

expressing the commensurability between the period $L$ and the average distance between the particles.

In order to characterize the system, we define three different states of commensurability. (i) $S_{I}$ : when $p \in \mathbb{Z}$, which is a commensurate state; (ii) $S_{I I}$ : when $p=k / m: k \in \mathbb{Z}, m \in \mathbb{N}$, $k \neq m$, it means that the system is not completely commensurate, this state could be subdivided into $S_{I I}^{-}$for $p<1$ and $S_{I I}^{+}$ for $p>1$; (iii) $S_{I I I}$ : when $p \in\{\mathbb{R}-\mathbb{Q}\}$ and it implies that the system is incommensurate. In this work we will limit our analysis to systems belonging to the first two categories.

Different experimental works $[19,37,40]$ have shown the feasibility of confining magnetic particles in a periodic potential by using lasers and optical tweezers, controlling the periodicity and strength of the potential, and also to manipulate the orientation of the magnetic field. These experimental results showed stable configurations even at room temperature.

We investigate the ground-state configuration of the system, using Monte Carlo (MC) simulations optimized with the Newton method, which has been previously used to analyze the structural transitions in Q1D systems with purely repulsive interaction [4-6,41]. This method allows us to calculate the eigenfrequencies and eigenmodes of the lattice vibrations for a given phase. Previous works [4,5] demonstrated that the lowest eigenfrequency is a measure of the stability of a configuration.

We will study the dependence of the ground-state configurations on the orientation of the magnetic field. For that, we fix the linear density of the system to the value $\eta=0.8$. Previously $[4,5,41]$ it was shown for B perpendicular to the $x-y$ plane that the dipoles are no longer arranged in a single-chain configuration at the center of the parabolic channel for $\eta=0.8$, but split into a two-chain configuration. The effect of the magnetic field strength will not be studied, since we are considering a system with superparamagnetic dipole particles, (i.e., the magnetic moment of all particles is aligned along B), therefore, all numerical results of the present work are calculated using $v=1$ and $\mu=1$.

\section{COMMENSURATE SYSTEM $(p=1)$}

For a typical commensurate state $(p=1)$, the anisotropy of the system is strongly determined by the direction of $\mathbf{B}$. In order to understand this effect, Fig. 3 shows the phase diagram of the system as a function of the planar $(\varphi)$ and azimuthal $(\theta)$ angles of the direction of $\mathbf{B}$, when the strength of the periodic potential is $V_{0}=0.03$. In this diagram the solid and dashed curves represent first- and second-order phase transitions, respectively.

In Fig. 4 we present the ground-state configurations as they are numbered in the phase diagram (see Fig. 3). The small horizontal red and vertical blue arrows indicate the displacement of the particles when increasing $\varphi$ and $V_{0}$, respectively, while the thick gray arrows between two configurations indicate that the transition between them occurs continuously.

From Fig. 4 one can see that, until phase 5, all ground-state configurations follow the following rule: one particle per one cell (we call cells the wells formed by the total confinement potential, which are marked in Fig. 4 by gray ellipses). However, with the exception of phase 1 , when increasing $\varphi$ most of the particles move away from the center of the cell due to the anisotropic interparticle interaction, following the directions shown by the horizontal red arrows.

\section{A. Transition from isotropy to anisotropy interaction}

From Eq. (3) we notice that for $\theta=0^{\circ}$ the interaction between particles is purely repulsive and is given by $V_{\text {dip }}(r)=$ $\mu^{2} / r^{3}$. As was found in Refs. [4,5,41], the ground state of the system is given by a zigzag configuration, which is represented by phase 2 . Notice also from Fig. 3 , that for $\theta=0^{\circ}$ the ground state is a zigzag configuration irrespective of the value of $\varphi$, evidencing the isotropy of the interparticle interaction.

The anisotropy of the interaction arises slowly by increasing $\theta$, resulting in a lowering of the lowest eigenfrequency of the

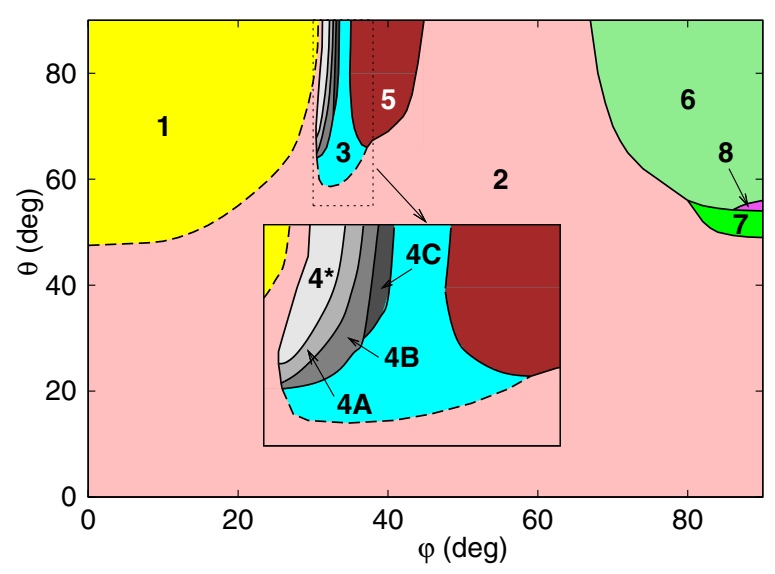

FIG. 3. (Color online) Phase diagram of the ground-state configuration, for a system of dipole particles in the regime $S_{I}$ with $p=1$ and $V_{0}=0.03$. The solid (dashed) lines represent first-order (second-order) transitions. Numbered phases are shown in Fig. 4. 

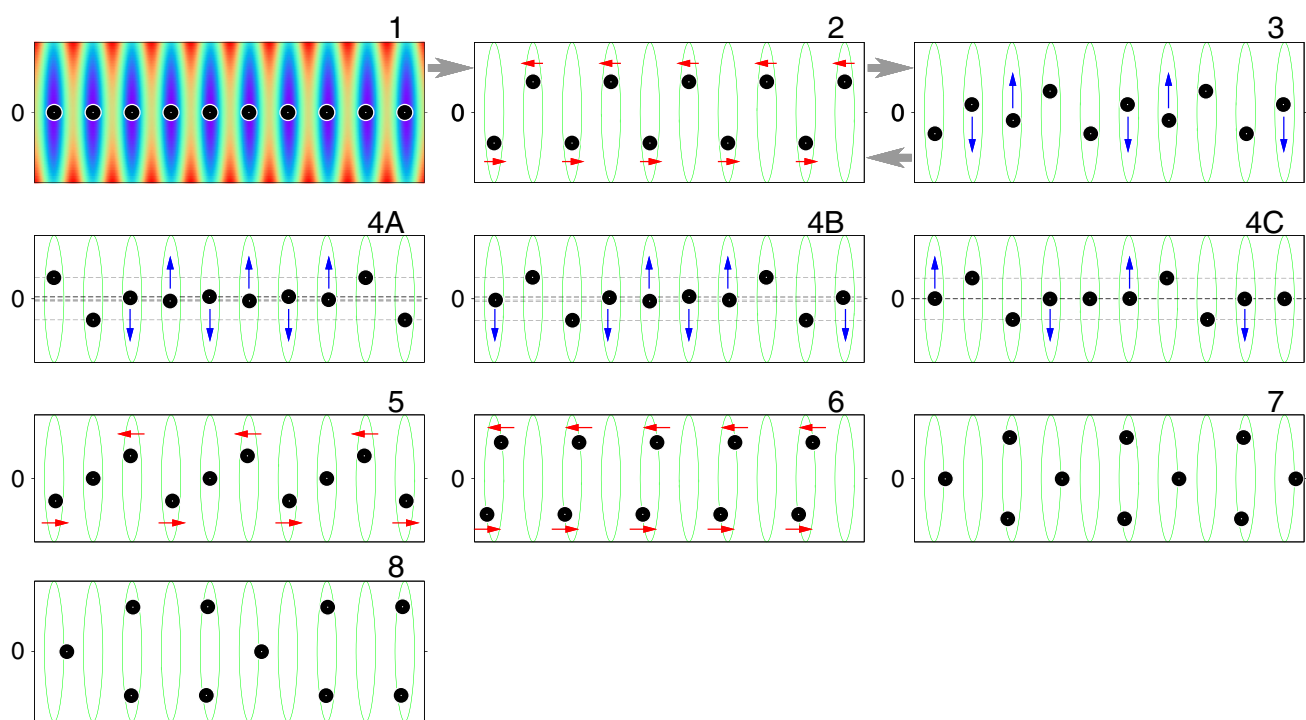

FIG. 4. (Color online) Different ground-state configurations found for the system with $p=1$. The small horizontal red (vertical blue) arrows show the movement of the particles in each phase caused by a small increment of $\varphi\left(V_{0}\right)$, while the dashed lines are a guide to the eye to show more clearly the different chains and the green ellipses represent the cells formed by the confining and periodic potentials (which is shown as a color contour plot in phase 1).

system for small $\varphi$ and it slowly increases with $\varphi$ as seen in Fig. 5. Taking into account that the stability of a configuration is directly related to the value of its lowest eigenfrequency, we notice, that as an effect of the anisotropy of the pairwise interaction, the stability of the two-chain configuration (phase 2 in Fig. 4) increases for intermediate values of $\varphi\left(45^{\circ} \lesssim \varphi \lesssim\right.$ $70^{\circ}$ ) by increasing $\theta$, while the opposite behavior was found for $\theta \lesssim 30^{\circ}$.

From Fig. 3 one can see that for $\theta \gtrsim 47^{\circ}$ the anisotropy of the pairwise interaction introduces new phases as ground state, now depending on $\varphi$. The very rich variety of phases produces in most of the cases first-order transitions between them, as is evidenced in Fig. 6, through the discontinuous jumps in the lowest eigenfrequency of the system.

The transition between the single- and the two-chain (phase 2) configuration (zigzag transition) appears in the region where

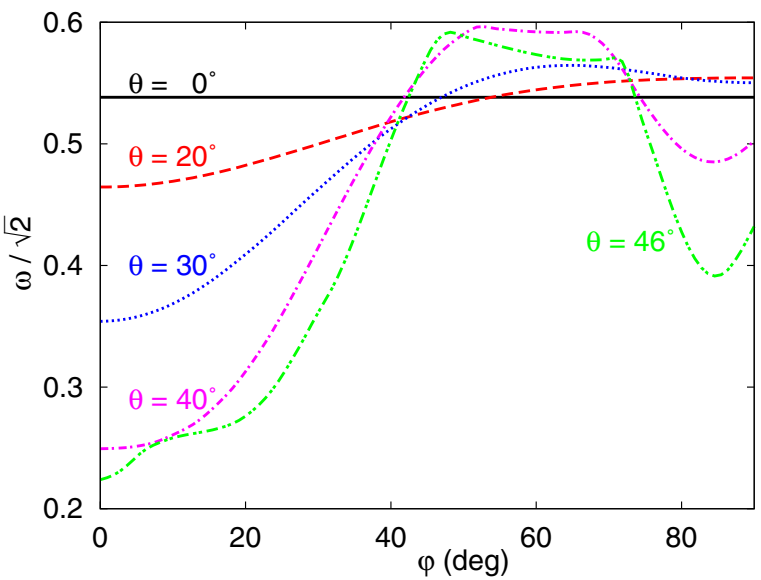

FIG. 5. (Color online) Lowest eigenfrequency of the two-chain configuration (phase 2) as a function of the planar angle $(\varphi)$ for different values of $\theta$. The other parameters are $p=1$ and $V_{0}=0.03$. the dipole-dipole interaction is dominantly attractive. For $\theta \gtrsim$ $47^{\circ}$, the single-chain configuration (phase 1 ) is the ground state for small values of $\varphi$ and this region increases with increasing $\theta$. The continuous transition between these two phases in the $y$ direction, is shown in Fig. 6(a), while Fig. 6(b) shows that the lowest eigenfrequency goes to zero which is the evidence of a second-order phase transition [4].

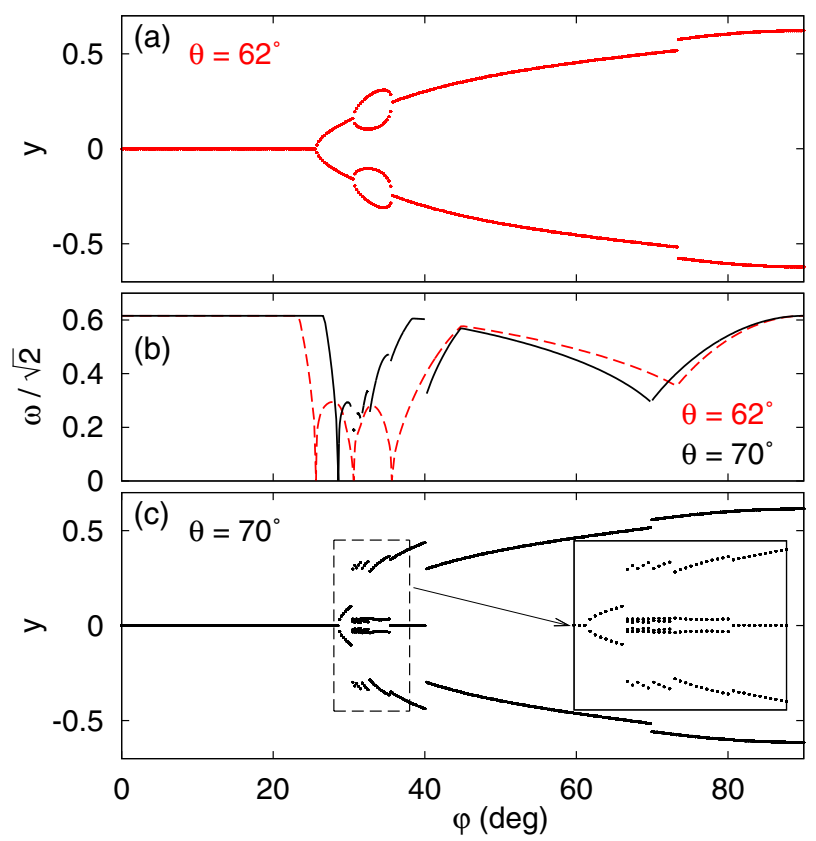

FIG. 6. (Color online) (a), (c) The $y$ position of the particles in the ground state for a system with $p=1$ and $V_{0}=0.03$ as a function of $\varphi$, for two different values of $\theta$ indicated in each figure. (b) Lowest eigenfrequency of the system for $\theta=62^{\circ}$ (dashed red line) and $\theta=$ $70^{\circ}$ (solid black line). Structural transitions occur when $\omega$ exhibits a jump (first order) or when it becomes zero (second order). 

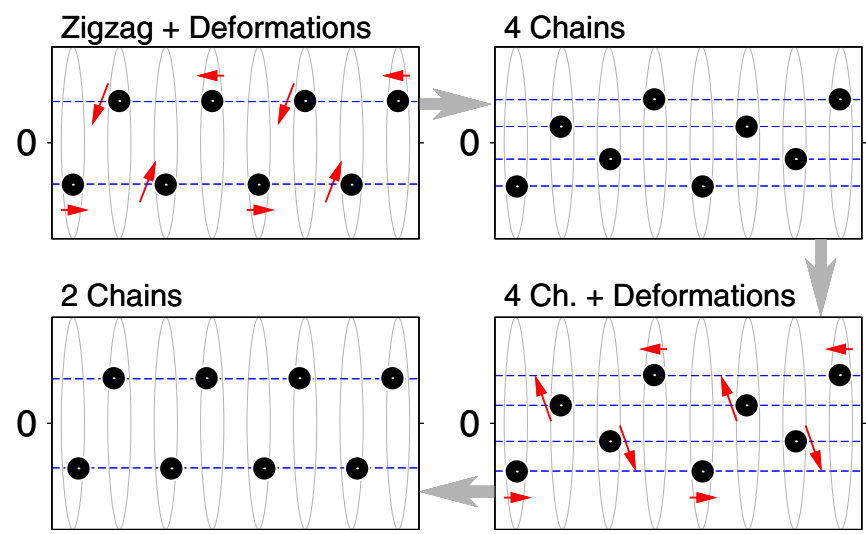

FIG. 7. (Color online) Mechanism of the reentrant process between $2-4-2$ chains in the system. The horizontal dashed lines accentuate the chainlike configuration of each phase.

\section{B. Transition between $2-4-2$ chains: Reentrant behavior}

A second-order transition in the ground-state configuration occurs between phases 2 and 4 (Fig. 4). Such a transition reveals an interesting effect, which is put in evidence in Fig. 6(a). The transition between $2-4-2$ chains is found by increasing $\varphi$ within the interval $\left(59^{\circ} \lesssim \theta \lesssim 63^{\circ}\right)$ in the $\theta-\varphi$ phase diagram (Fig. 3). This is demonstrated in Fig. 6(a) for $\theta=62^{\circ}$. A similar reentrant process has been found in dipolar fluids [42] and recently also for Q1D systems of patchy particles [43,44] and ferrogels [45].

This reentrant process occurs after the zigzag transition takes place. In Fig. 6(b) we show that, by increasing $\varphi$, the two-chain configuration (phase 2) becomes less stable, since its lowest frequency approaches zero at the point where the four-chain configuration (phase 3) arises from a continuous lattice deformation of phase 2 . After that, the stability of the four-chain configuration increases until $\varphi \simeq 33^{\circ}$, where the lowest eigenfrequency starts to decrease, returning to the twochain configuration through a continuous deformation of the lattice. During this process the lowest eigenfrequency of phase 3 decreases to zero, after that phase 2 appears again through a second-order transition [Fig. 6(b)].

The mechanism of this reentrant process, which occurs through a continuous deformation of the lattice, is outlined in Fig. 7. Notice that this reentrant process is qualitatively different from the one found earlier in Ref. [4], where these processes were found to be of first order. This difference is due to the presence of the periodic potential. Without the periodic potential the particles not only undergo a zigzag transition, but they also exhibit a discontinuous shift in the $x$ direction, which now is prevented by the periodic potential.

\section{Solitonlike deformations of the single-chain phase}

For larger values of $\theta\left(\theta \gtrsim 70^{\circ}\right)$ the anisotropy of the dipoledipole interaction determines most of the transitions found for the ground-state configurations. It forces the particles along B to align forming (short) rowlike arrangements, which may even result in the localization of two particles in the same cell of the periodic potential, as shown in Fig. 3 for $V_{0}=0.03$ (e.g., see phases 2, 5, and 6). Some of these transitions result in a sequential deformation of the single-chain configuration as shown in the highlighted region in Fig. 6(c), where the $y$ position of the particles is plotted as a function of $\varphi$ for $\theta=70^{\circ}$. In this case the deformations appear between the two- and four-chain configurations, and the region between the phases is identified in the phase diagram (Fig. 3) as phase 4.

In Fig. 6(c) one can observe that by increasing $\varphi$ the ground-state phase transitions in the highlighted region are a sequence of single-chain and two-chain configurations followed by a group of disorderlike phases and after that fourand three-chain configurations, where all transitions are of first order with the exception of the zigzag transition, which has been previously analyzed. The first-order transitions are evidenced by the discontinuities in the lowest eigenfrequency of the system [4,6] and the jumps in the $y$-coordinate position.

The disorderlike phases aforementioned are a consequence of the high anisotropy of the interaction potential. In order to understand this effect we consider the limiting case $\theta=90^{\circ}$, where the dipole-dipole interaction is dominantly attractive for $\varphi<30^{\circ}$. In this limit the competition between the interparticle interaction and the periodic confinement potential $\left(V_{0}=0.03\right)$ produces a deformation of the single-chain configuration immediately after the two-chain configuration appears. The single-chain configuration is suddenly broken by a local deformation of the lattice, after that, the period of the position where such a deformation occurs decreases with $\varphi$ until the four-chain configuration (phase 3 ) is reached.

In the upper part of Fig. 8 we show the deformation process presenting some configurations that are typical for phase $4^{*}$.

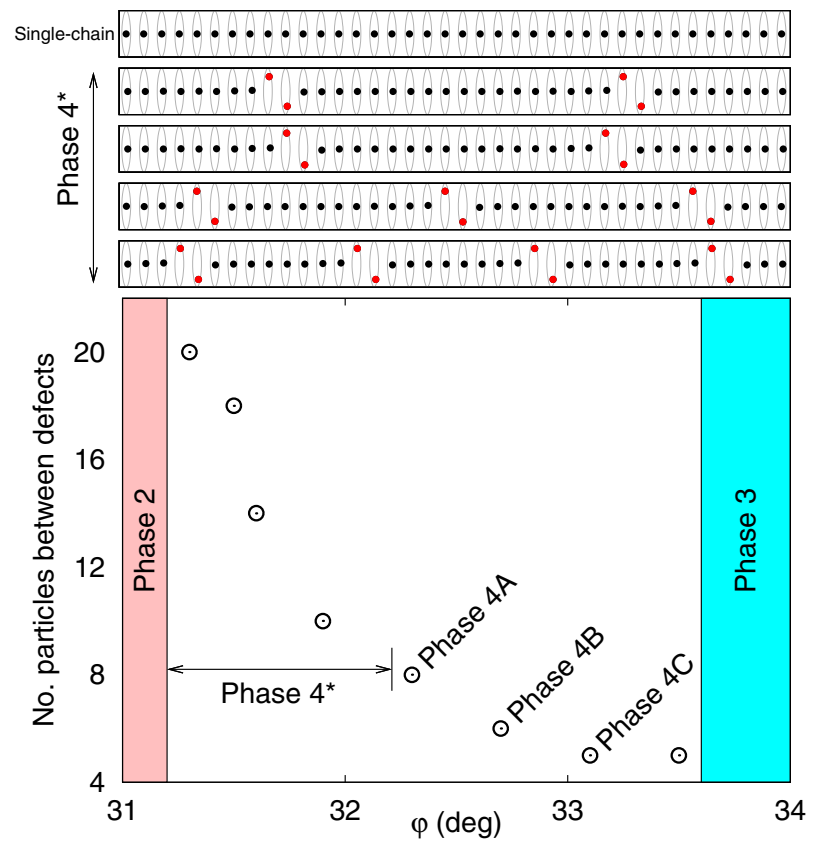

FIG. 8. (Color online) The number of particles between defects (i.e., solitons) during the single-chain sequential deformation process, is plotted as a function of $\varphi$ for $\theta=90^{\circ}$ and $V_{0}=0.03$. Configurations of the disordered phases are shown in the upper figures, where the positions of the defects are highlighted by red symbols for the particles, and the gray ellipses indicate the cells formed by the confinement in combination with the periodic potential. 
In those configurations the location of the defects are marked with red symbols for the particles. Similar defects have been experimentally found in Q1D zigzag configurations of ion crystals and were reported recently in Refs. [46-49], where they are considered as solitonlike configurations. Defects appear as frozen solitons whose density increases with $\varphi$. From those configurations, the deformation process can be understood as a perturbation of the one-dimensional lattice, uniformly distributed along the axial direction, which allows the system to evolve through first-order transitions into a Wigner crystalline structure. In order to clarify this process, we plot the number of particles between defects (or solitons) as a function of $\varphi$ for the largest anisotropy of the interaction between particles (i.e., $\theta=90^{\circ}$ ) when $V_{0}=0.03$, in Fig. 8 . This figure shows how the spatial frequency of the defects (or the density of solitons) increases by increasing $\varphi$, reducing the number of particles between defects until the groundstate structure reaches the four-chain configuration (phase 3). This process has been found previously for an infinite system of particles interacting through a purely repulsive interaction, confined by a power-law potential $y^{\alpha}$ when $\alpha<2$, by increasing the linear density of the system [5].

\section{Effect of the periodic substrate potential}

In this section we pay attention to the effect of the substrate potential on the ground-state configuration in the commensurate case with $p=1$ and $\theta=90^{\circ}$. The groundstate configurations are summarized in the $V_{0}-\varphi$ diagram, which is presented in Fig. 9. Notice that for $\theta=90^{\circ}$ the anisotropy of the dipole-dipole interaction is maximum (see Fig. 2). For some intervals of $\varphi$ (e.g., $\varphi \lesssim 30^{\circ}, \varphi \gtrsim 68^{\circ}$ ) the ground-state configuration is unaffected by $V_{0}$. For $\varphi \lesssim 30^{\circ}$ the dipole-dipole interaction along the $x$ axis is dominantly attractive and the single-chain configuration is found as the ground state (Fig. 4). On the other hand, for $\varphi \gtrsim 60^{\circ}$ the

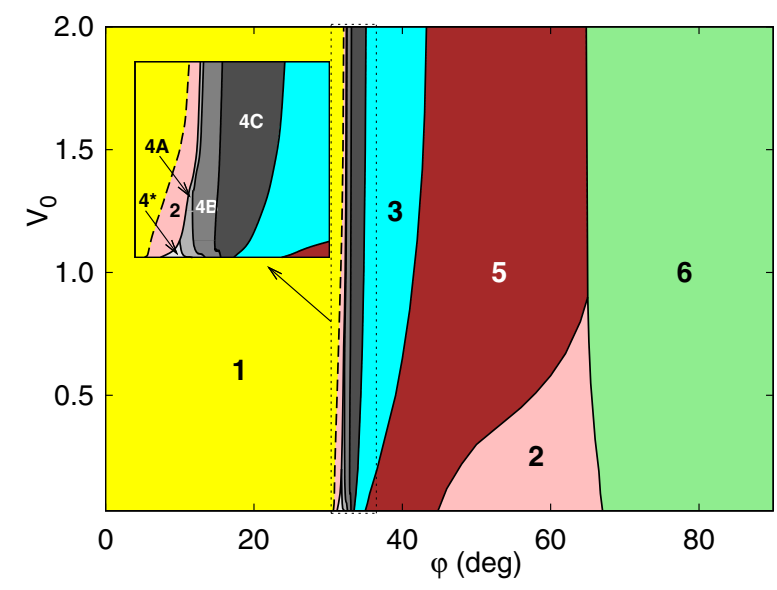

FIG. 9. (Color online) Phase diagram of the ground-state configuration, for a system of dipole particles for the case $S_{I}$ with $p=1$ and $\theta=90^{\circ}$. Different phases are plotted in different colors as a function of the angle $\varphi$ and the strength of the substrate potential $V_{0}$. The solid (dashed) lines represent first-order (second-order) transitions between the phases. Numbered phases are shown in Fig. 4. The dashed box area is enlarged in the inset. dipole-dipole interaction along the $x$ axis becomes dominantly repulsive and the two-chain configuration is now obtained as the ground state, which is independent of $V_{0}$. There is an intermediate interval $30^{\circ} \lesssim \varphi \lesssim 68^{\circ}$ in which it is possible to control the system configuration through the strength of the substrate $V_{0}$.

The process of the single-chain sequential deformation, as previously discussed, is attenuated by increasing $V_{0}$ reducing this process to a very narrow region (see inset in Fig. 9), where the deformation is reduced to the phases $4 A-4 C$. For $V_{0}>1$ the ground-state configuration is less sensitive to a variation of $V_{0}$, as is visible in Fig. 9. In all cases the effect of $V_{0}$ is to rearrange the configuration by moving the particles towards the center of the cell in the $y$ direction, as was previously studied in Ref. [35].

It is interesting to note that phases 7 and 8 do not appear in Fig. 9, showing that these are characteristic configurations produced by the anisotropy of the interaction, which can be found in a small window of the angles $\theta$ and $\varphi$, for a weak strength of the periodic potential $V_{0}$, as shown in Fig. 3. The configuration of these phases (see Fig. 4) is evidence that the position of the particles are mainly determined by the interaction potential.

\section{NONCOMPLETELY COMMENSURATE $\operatorname{SYSTEM}(p=1 / 2)$}

We further analyze the influence of the commensurability factor on the system by taking $p=1 / 2$ bearing in mind that $p$ can be controlled by $L$ or the linear density $\eta$ [see Eq. (5)]. The ground-state configurations are summarized in the $V_{0}-\varphi[\theta-\varphi]$ phase diagram for $\theta=90^{\circ}\left[V_{0}=0.03\right]$ which is presented in Fig. 10(a) [10(b)]. The configurations of the numbered phases in Fig. 10 are shown in Fig. 11, where the gray ellipses indicate the cells formed by the confinement and the periodic potential.

From Fig. 10(a), one can see that the effect of the periodic potential increases with increasing $V_{0}$, as was studied in previous section, and it results in a rearrangement of the configuration, moving the particles towards the center of the cell. The single-chain sequential deformation process described by phases $4^{*}$ and $4 A-4 C$, which were analyzed in Sec. III C, is still present for $p=1 / 2$. However, due to the existence of an empty cell between two nearest particles in these phases (see Fig. 11), the movement in the $x$ direction is highly restricted, as a consequence the single-chain deformation process will be reduced rapidly to the phases $4 B$ and $4 C$ by increasing $V_{0}$, as is shown by the inset in Fig. 10(a). In the same way, the zigzag transition disappears around $V_{0}=0.5$, allowing the system to transit from phase 1 directly to phase 4 for higher values of $V_{0}$.

On the other hand, Fig. 10(b) shows that due to the symmetry of the periodic potential wells, the effect of the interaction anisotropy on the system for small values of $\theta$, is similar to the previously discussed case of $p=1$ allowing to make the two-chain configuration (phase 2 in Fig. 11) as the ground state. Additionally, by increasing $\theta$ the stability of phase 2 decreases allowing the system to reach other configurations as ground state. After that, several phases are found, but only until phase 5 the ground-state 


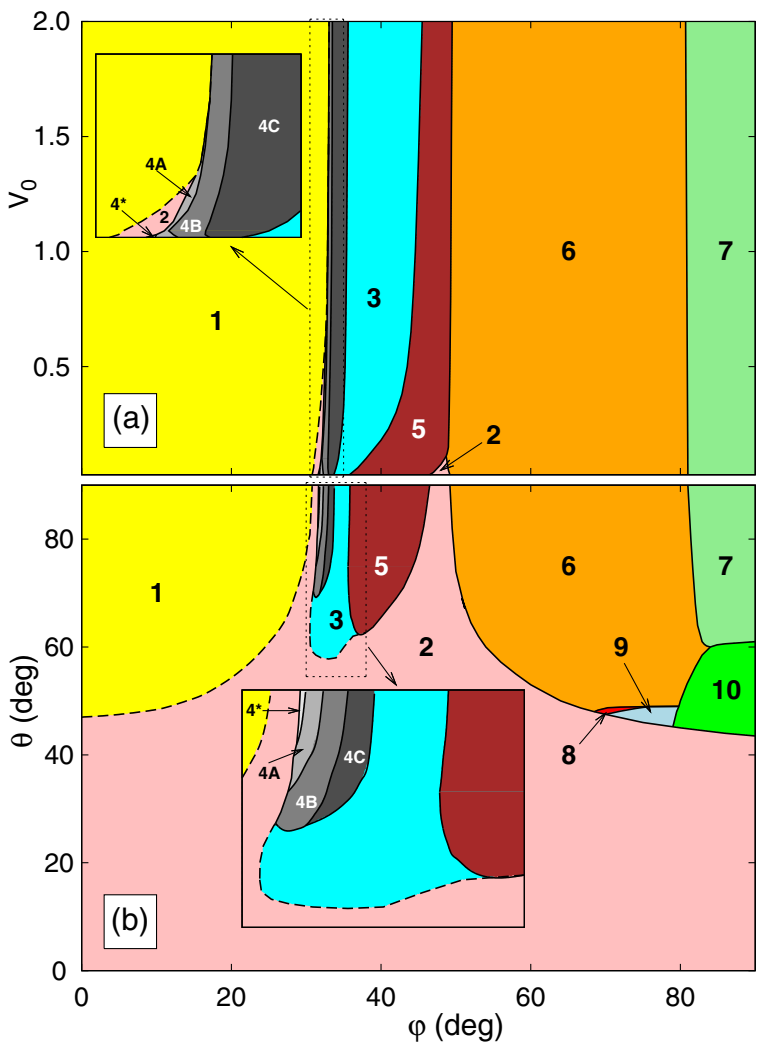

FIG. 10. (Color online) Phase diagram of the ground-state configuration for a system of dipole particles in the regime $S_{I I}$ with $p=1 / 2$. Different phases are plotted as a function of (a) $\varphi$ and $V_{0}$ for $\theta=90^{\circ}$, and (b) $\varphi$ and $\theta$ for $V_{0}=0.03$. The solid (dashed) lines represent first-order (second-order) transitions between phases. Numbered phases are shown in Fig. 11. The dashed box areas are elongated in the insets. configurations correspond to one particle per two cells, showing the large influence of $p$ on the system inside the region $\varphi<50^{\circ}$.

As a consequence of the new symmetry imposed by the commensurability factor some new configurations, beyond those found for the case $p=1$, are found for the case $p=1 / 2$. As was discussed at the end of previous section, these configurations (phases 6, 8, and 9) are generated as a consequence of the anisotropy of the interaction potential for intermediate values of $\theta$.

Figures 12(a) and 12(b) show an interesting transition in the region $60^{\circ} \lesssim \varphi \lesssim 80^{\circ}$ for $\theta=48^{\circ}$ and $V_{0}=0.03$. In this region the ground state changes between phases $2-6-8-9-10$, where the first two correspond to different two-chain configurations, and the last one is a typical Q1D Wigner crystal with a three-chain configuration. Nevertheless, transition between phase 8 (eight chains) and phase 9 (ten chains) occurs with a small discontinuity in its lowest eigenfrequency as shown in Fig. 12(b) around $\varphi=71^{\circ}$. Such a transition is even more clear in Fig. 12(a) where the $y$ position of the particles are plotted as a function of $\varphi$. In Fig. 11, the configuration of phases 8 and 9 are shown where the dashed lines indicate the position of the different chains. Additionally, the red arrows show the displacement of the particles when $\varphi$ increases.

The two-chain configuration for $p=1 / 2$ is found in three different phases (2, 6, and 7 in Fig. 11), which shows that the zigzag symmetry is broken with increasing $\varphi$. However, the transition between these phases is discontinuous, even for small values of $V_{0}$, as shown in Fig. 12(b) for $\theta=66^{\circ}$ and $V_{0}=$ 0.03 , where the discontinuities in the lowest eigenfrequency of the system indicate first order transitions between these phases. These discontinuities are also evident in the $y$ position of the particles as a function of $\varphi$ in Fig. 12(c).
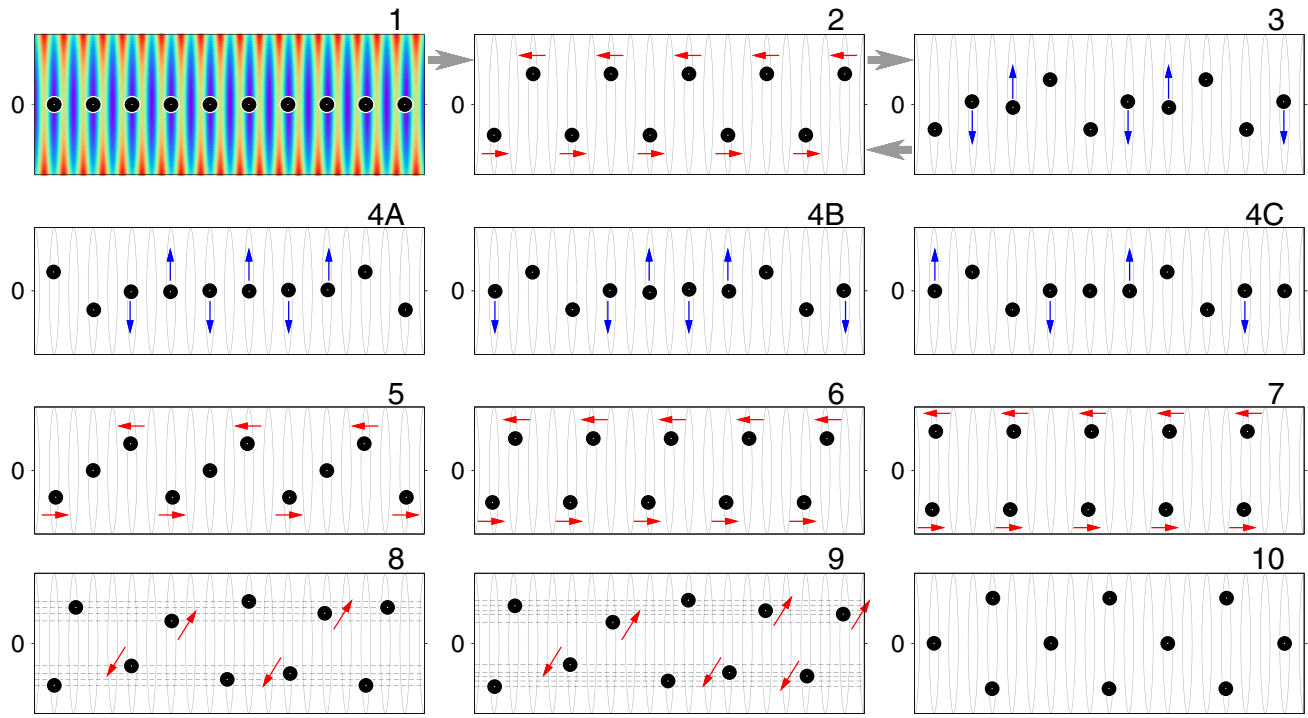

FIG. 11. (Color online) Different ground-state configurations found for the system with $p=1 / 2$. The small horizontal and diagonal red (vertical blue) arrows show the movement of the particles in each phase caused by a small increment of $\varphi\left(V_{0}\right)$, while the dashed lines accentuate the different chains and the gray ellipses represent the cells formed by the confining and periodic potentials (which is shown as a color contour plot in phase 1). 

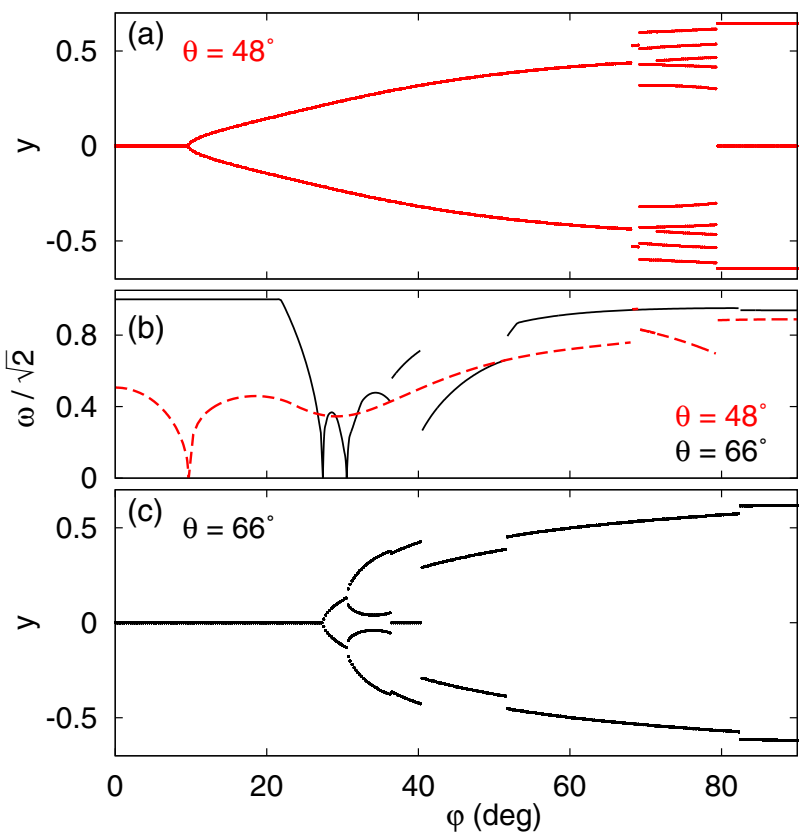

FIG. 12. (Color online) (a), (c) The $y$ position of the particles in the ground state for a system with $p=1 / 2$ and $V_{0}=0.03$ as a function of $\varphi$, for two different values of $\theta$ indicated in each figure. (b) Lowest eigenfrequency of the system for $\theta=48^{\circ}$ (dashed red line) and $\theta=66^{\circ}$ (solid black line).

\section{CONCLUSIONS}

We studied a Q1D system of magnetic particles confined by a parabolic channel modulated by a periodic substrate potential, where the magnetic moment of all particles is aligned by an external tilted magnetic field. The linear density in the direction of the modulated potential was fixed to $\eta=0.8$ and the ground-state configuration at zero temperature was analyzed as a function of the magnetic field orientation, strength of the periodic potential and commensurability factor. A plethora of different particle configurations were found as ground state, which are arranged in a different number of chains, and we even found a remarkable solitonlike configuration where the density of solitons could be varied with the tilt angle.

The anisotropy of the pairwise interaction between particles, determined by the magnetic tilt angles $\theta$ and $\varphi$ is largely responsible for the crystalline configuration of the system, but its effect decreases by increasing $V_{0}$. For small values of $V_{0}$, the angle $\theta$ controls the degree of anisotropy of the system and with the in-plane angle $\varphi$ it is possible to tune the ground-state configuration. On the other hand, for the limiting case of maximum pairwise anisotropy $\left(\theta=90^{\circ}\right)$, the control of the ground-state configuration depends weakly on $\varphi$ for large values of $V_{0}$.

The commensurability factor not only changes the stability region of the phases, as shown in the phase diagrams, but it also produces the emergence of characteristic ground-state configurations due to its symmetry. However, in order to get these characteristic phases for each commensurability factor, it is necessary to tune $\theta$ and $\varphi$ to appropriate values.

From these results we can conclude that varying $\theta$ and $\varphi$ allows us to tune the ground state of the system, while the effect of $V_{0}$ is to rearrange the configuration by moving the particles to the center of the cells in the $y$ direction. The commensurability factor, controlled by $L$ or the density, acts as a complementary tunable parameter, which gives the freedom to build a desired configuration.

The large variety of chainlike phases found in this work allows us to suggest that the effects of the parameters on the system will be similar for larger values of the density, where the number of phases may increase but the transition between them will be qualitatively similar. This could be very useful as input parameter for experiments with magnetic particles, where the sinusoidal confinement can be realized by the application of external potentials through spatially varying light fields, which have been used to induce structural changes in colloidal systems $[13,33,50]$, while the configurations can be obtained by video microscopy for different external magnetic fields as was demonstrated in Ref. [16].

\section{ACKNOWLEDGMENTS}

This work was supported by the Flemish Science Foundation (FWO-Vl), the Methusalem programme of the Flemish government, CNPq, CAPES, FUNCAP (Pronex grant), the collaborative program CNPq - FWO-Vl, and the Brazilian program Science Without Borders CsF. Computational resources were provided by HPC infrastructure of University of Antwerp (CalcUA) a division of the Flemish Supercomputer Center (VSC).
[1] E. V. Shevchenko, D. V. Talapin, N. A. Kotov, S. O'Brien, and C. B. Murray, Nature (London) 439, 55 (2006).

[2] Y. Min, M. Akbulut, K. Kristiansen, Y. Golan, and J. Israelachvili, Nature Mater. 7, 527 (2008).

[3] R. M. Choueiri, A. Klinkova, H. Thérien-Aubin, M. Rubinstein, and E. Kumacheva, J. Am. Chem. Soc. 135, 10262 (2013).

[4] G. Piacente, I. V. Schweigert, J. J. Betouras, and F. M. Peeters, Phys. Rev. B 69, 045324 (2004).

[5] J. E. Galván-Moya and F. M. Peeters, Phys. Rev. B 84, 134106 (2011).

[6] J. E. Galván-Moya, K. Nelissen, and F. M. Peeters, Phys. Rev. B 86, 184102 (2012).
[7] V. A. Schweigert and F. M. Peeters, Phys. Rev. B 51, 7700 (1995).

[8] B. Partoens, V. A. Schweigert, and F. M. Peeters, Phys. Rev. Lett. 79, 3990 (1997).

[9] R. W. Hasse and V. V. Avilov, Phys. Rev. A 44, 4506 (1991).

[10] Y. G. Cornelissens, B. Partoens, and F. M. Peeters, Physica E 8, 314 (2000).

[11] C. J. Olson Reichhardt, C. Reichhardt, and A. R. Bishop, Phys. Rev. Lett. 92, 016801 (2004).

[12] Y. H. Liu, L. Y. Chew, and M. Y. Yu, Phys. Rev. E 78, 066405 (2008). 
[13] J. Drocco, L. M. Lopatina, C. Reichhardt, and C. J. OlsonReichhardt, Proc. SPIE 8458, 84581I (2012).

[14] H. J. Zhao, V. R. Misko, and F. M. Peeters, New J. Phys. 14, 063032 (2012).

[15] V. A. Froltsov, R. Blaak, C. N. Likos, and H. Löwen, Phys. Rev. E 68, 061406 (2003).

[16] H. Löwen, R. Messina, N. Hoffmann, C. N. Likos, C. Eisenmann, P. Keim, U. Gasser, G. Maret, R. Goldberg, and T. Palberg, J. Phys.: Condens. Matter 17, S3379 (2005).

[17] A. Reinmüller, E. C. Oguz, R. Messina, H. Löwen, H. J. Schöpe, and T. Palberg, Eur. Phys. J. Special Topics 222, 3011 (2013).

[18] S. Deutschländer, T. Horn, H. Löwen, G. Maret, and P. Keim, Phys. Rev. Lett. 111, 098301 (2013).

[19] H. Löwen, Eur. Phys. J. Special Topics 222, 2727 (2013).

[20] V. Danilov, T. Prokopyeva, and S. Kantorovich, Phys. Rev. E 86, 061408 (2012).

[21] S. Jager and S. H. L. Klapp, Phys. Rev. E 86, 061402 (2012).

[22] D. Lucena, W. P. Ferreira, F. F. Munarin, G. A. Farias, and F. M. Peeters, Phys. Rev. E 87, 012307 (2013).

[23] M. Knap, E. Berg, M. Ganahl, and E. Demler, Phys. Rev. B 86, 064501 (2012).

[24] A. G. Volosniev, J. R. Armstrong, D. V. Fedorov, A. S. Jensen, and N. T. Zinner, Few-Body Syst. 54, 707 (2013).

[25] B. Wunsch, N. T. Zinner, I. B. Mekhov, S. J. Huang, D. W. Wang, and E. Demler, Phys. Rev. Lett. 107, 073201 (2011).

[26] J. Ruhman, E. G. Dalla Torre, S. D. Huber, and E. Altman, Phys. Rev. B 85, 125121 (2012).

[27] B. Spivak and S. A. Kivelson, Phys. Rev. B 70, 155114 (2004).

[28] M. Bauer and M. M. Parish, Phys. Rev. Lett. 108, 255302 (2012).

[29] Y. Yang, L. Gao, G. P. Lopez, and B. B. Yellen, ACS Nano 7, 2705 (2013).

[30] J. Fornleitner, F. L. Verso, G. Kahl, and C. N. Likos, Soft Matter 4, 480 (2008).

[31] J. Fornleitner, F. L. Verso, G. Kahl, and C. N. Likos, Langmuir 25, 7836 (2009).

[32] A. Chremos and C. N. Likos, J. Phys. Chem. B 113, 12316 (2009).

[33] R. D. L. Hanes, C. Dalle-Ferrier, M. Scmiedeberg, M. C. Jenkins, and S. U. Egelhaaf, Soft Matter 8, 2714 (2012).
[34] J. C. N. Carvalho, K. Nelissen, W. P. Ferreira, G. A. Farias, and F. M. Peeters, Phys. Rev. E 85, 021136 (2012).

[35] J. C. N. Carvalho, W. P. Ferreira, G. A. Farias, and F. M. Peeters, Phys. Rev. B 83, 094109 (2011).

[36] T. Neuhaus, M. Schmiedeberg, and H. Löwen, New J. Phys. 15, 073013 (2013).

[37] L. D. Carr, D. DeMille, R. V. Krems, and J. Ye, New J. Phys. 11, 055049 (2009).

[38] S. Herrera-Velarde and R. Castaneda-Priego, J. Phys.: Condens. Matter 19, 226215 (2007).

[39] C. Reichhardt and C. J. Olson Reichhardt, Phys. Rev. E 85, 051401 (2012).

[40] C. Dalle-Ferrier, M. Kruger, R. D. L. Hanes, S. Walta, M. C. Jenkins, and S. U. Egelhaaf, Soft Matter 7, 2064 (2011).

[41] G. Piacente, G. Q. Hai, and F. M. Peeters, Phys. Rev. B 81, 024108 (2010).

[42] T. Tlusty and S. A. Safran, Science 290, 1328 (2000).

[43] Y. Yang, L. Gao, G. P. Lopez, and B. B. Yellen, J. Chem. Phys. 135, 034501 (2011).

[44] S. Roldán-Vargas, F. Smallenburg, W. Kob, and F. Sciortino, J. Chem. Phys. 139, 244910 (2013).

[45] M. A. Annunziata, A. M. Menzel, and H. Löwen, J. Chem. Phys. 138, 204906 (2013).

[46] S. Ulm, J. Roßnagel, G. Jacob, C. Degünther, S. T. Dawkins, U. G. Poschinger, R. Nigmatullin, A. Retzker, M. B. Plenio, F. Schmidt-Kaler, and K. Singer, Nature Commun. 4, 2290 (2013).

[47] K. Pyka, J. Keller, H. L. Partner, R. Nigmatullin, T. Burgermeister, D. M. Meier, K. Kuhlmann, A. Retzker, M. B. Plenio, W. Zurek, A. del Campo, and T. E. Mehlstäubler, Nature Commun. 4, 2291 (2013).

[48] H. L. Partner, R. Nigmatullin, T. Burgermeister, K. Pyka, J. Keller, A. Retzker, M. B. Plenio, and T. E. Mehlstäubler, New J. Phys. 15, 103013 (2013).

[49] H. Landa, B. Reznik, J. Brox, M. Mielenz, and T. Schaetz, New J. Phys. 15, 093003 (2013).

[50] C. Bechinger, M. Brunner, and P. Leiderer, Phys. Rev. Lett. 86, 930 (2001). 